\title{
Improving the resolution of dynamic intensity modulated radiation therapy delivery by reducing the multileaf collimator sampling distance
}

\author{
Peter B. Greer, ${ }^{\text {a) }}$ Wayne A. Beckham, and William Ansbacher \\ B.C. Cancer Agency, Vancouver Island Center, Victoria, B.C., Canada \\ Rita K. Mann \\ University of Victoria, Victoria, B.C., Canada \\ (Received 10 October 2002; accepted for publication 17 July 2003; published 24 September \\ 2003)
}

The conformality of a dose distribution delivered by a multileaf collimator (MLC) for intensity modulated radiation therapy (IMRT) is limited in the direction perpendicular to leaf motion by the finite leaf width. Two methods of improving the resolution of IMRT intensity maps in this direction were investigated. In the first, the desired fluence distribution is considered to be sampled by the MLC, with the sampling distance being the center-to-center distance between the MLC leaves. The sampling distance is reduced below the leaf width by combining separate irradiations with a couch shift between them. This has been applied to static field therapy [Galvin et al., Int. J. Radiat. Oncol., Biol., Phys. 35, 89-94 (1996)], and was proposed for IMRT by Bortfeld et al. [Med. Phys. 27, 2494-2502 (2000)]. In the second method, two MLC component fluences, with leaf width $L$ $=2 \Delta y$ and offset by $\Delta y$, are combined to reproduce desired intensity bins with $\Delta y$ width. The effect of MLC leaf sampling distance on dose resolution was quantified for both 1.0 and $0.5 \mathrm{~cm}$ MLC leaf widths, utilizing a high resolution bar-pattern fluence, an annular shaped fluence, and an intensity step-edge. Improvement in resolution was found for the $1.0 \mathrm{~cm}$ leaf width at a sampling distance of $0.5 \mathrm{~cm}$, with only a small benefit for further reduction. For the $0.5 \mathrm{~cm}$ leaf width, a sampling distance of $0.25 \mathrm{~cm}$ resulted in a dose resolution that was nearly independent of direction. (C) 2003 American Association of Physicists in Medicine. [DOI: 10.1118/1.1609992]

Key words: resolution, intensity modulation, multileaf collimators, dose painting

\section{INTRODUCTION}

The resolution of an intensity distribution produced by a multileaf collimator (MLC) for intensity modulated radiation therapy (IMRT) is limited in the direction perpendicular to leaf motion by the finite leaf width. The most widely utilized MLCs have a leaf width of $1.0 \mathrm{~cm}$ at isocenter, although smaller leaf-widths have become available. This reduces the ability to accurately "paint dose" and ultimately deliver high dose to target regions and low dose to normal tissues.

Methods to improve the resolution of intensity maps with a $1.0 \mathrm{~cm}$ leaf width MLC have been proposed where two orthogonal intensity maps are delivered with $1.0 \times 0.5 \mathrm{~cm}$ resolution, to give a combined result with higher $(0.5$ $\times 0.5 \mathrm{~cm}$ ) resolution. ${ }^{1,2}$ These approaches require rotation of the collimator assembly by 90 degrees between the two field segments. A Varian CL21EX (Varian Palo Alto, CA) with multileaf collimator takes $\sim 25 \mathrm{~s}$ to rotate through $90^{\circ}$, making the orthogonal delivery method time-intensive in practice.

A method to improve the shaping of static (non-IMRT) field edges with MLC leaves has been described where the sampling distance of the MLC leaves is decreased. ${ }^{3,4}$ The sampling distance is the center-to-center distance between adjacent leaves. This is achieved by delivering a field as segments. For each segment the MLC leaves are offset by a fraction of the leaf width (in the direction perpendicular to leaf motion) and re-fitted to the field border. A couch shift is performed between each segment to offset the MLC leaves. In principle, dynamic multileaf collimator IMRT (DMLCIMRT) could also be delivered with this method. The time between each DMLC sequence would be limited by the time to reposition the couch. The MLC sequences could sweep in alternating directions and, therefore repositioning of the MLC between these sequences would be unnecessary.

Bortfeld et al. ${ }^{9}$ in their theoretical study examined the minimum MLC leaf sampling distance that is required to deliver arbitrary static and IMRT dose distributions. They proposed that the leaf width could be twice the sampling distance with a relatively minor deterioration in the dose. An experimental investigation of the effect of reducing the sampling distance on IMRT dose was not performed.

In this paper, two methods of improving the resolution of IMRT delivery are investigated. In the first method, the desired fluence distribution is considered to be sampled by the MLC. The sampling distance is decreased below the leaf width by multiple offset irradiations. In the second method, two offset irradiations are combined to attempt to reproduce the discrete intensity bins in the original fluence. In this case the bins in the original fluence are half the leaf width of the component deliveries. The effect of the MLC sampling distance on the dose resolution of DMLC-IMRT is experimentally investigated for both $1.0 \mathrm{~cm}$ and $0.5 \mathrm{~cm}$ MLC leaf widths. 


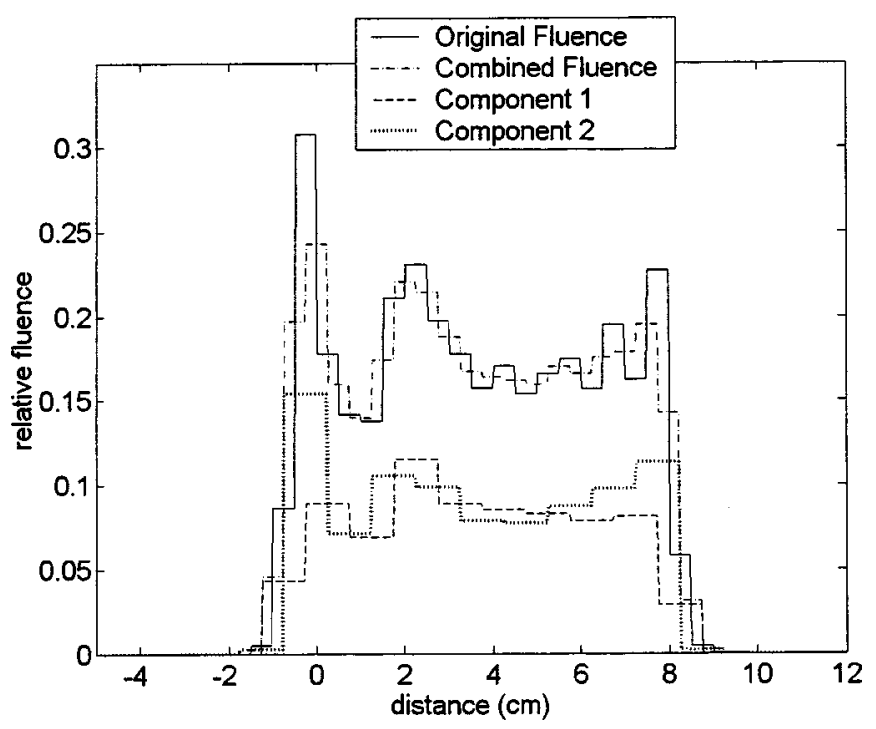

FIG. 1. Profile from a clinical fluence map in the $y$-direction across the leaves, and the two component fluence maps formed with a MLC leaf width of $L=1.0 \mathrm{~cm}$ and a sampling distance of $\Delta y=0.5 \mathrm{~cm}$. The resulting fluence is the average of the two adjacent bins in the original fluence.

\section{METHODS AND MATERIALS}

\section{A. Methods to improve the resolution of DMLC-IMRT}

\section{Sampling method}

The theoretical framework for reducing the MLC sampling distance is given in the Appendix. The theory outlined in the Appendix is based on a fluence function that is continuous. This is an approximation for fluence maps currently produced by the radiation therapy planning (RTP) systems that are generally at MLC leaf width spacing in the $y$-direction. In this work the $y$-direction is used to refer to the direction across the leaves, while the direction of leaf motion is the $x$-direction. Figure 1 shows a profile in the $y$-direction from an original (desired) clinical fluence map $\Phi(y)$ with $0.5 \mathrm{~cm}$ resolution derived by the RTP system optimization. The methodology outlined in the Appendix is applied to "sample" this fluence with an MLC leaf width of $L$ $=1.0 \mathrm{~cm}$ and a sampling distance of $\Delta y=0.5 \mathrm{~cm}$. Two MLC irradiations are required, delivered with a couch offset of $\Delta y=0.5 \mathrm{~cm}$. These component fluences are shown in Fig. 1 . Each MLC position is centered on the original fluence bin and hence offset from the original fluence bins by half the sampling distance. The fluence for each component irradiation is halved as the fluence bins overlap. To derive the separate MLC fluence maps is trivial in this case, as every second row of the original fluence map can be utilized. The resulting combined fluence is also shown in Fig. 1. These component maps would be input to the leaf-sequencer algorithm to produce MLC motion files and two deliverable fluence maps. The sampling distance can be reduced further. For example this could be reduced to $\Delta y=0.25 \mathrm{~cm}$ by utilizing four MLC irradiations offset by $0.25 \mathrm{~cm}$. The resolution of the desired fluence map would need to be increased to at least $0.25 \mathrm{~cm}$.

To calculate the dose-distribution that would result from this delivery, the RTPS would utilize the combined fluence

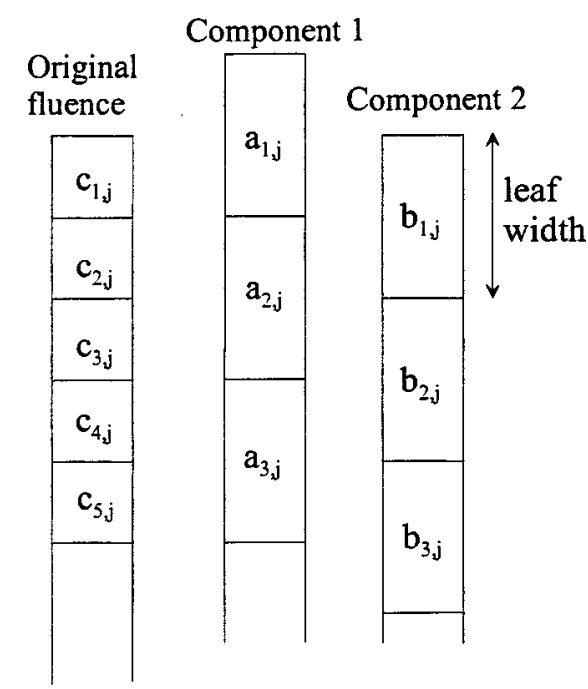

FIG. 2. Illustration of combining two component fluence maps, each with twice the leaf width of the desired intensity bins, to reproduce the desired intensity.

from the two deliverable fluence maps. This method is independent of the leaf-sequencing algorithm. The only proviso is that the resolution of the IMRT optimization and hence that of the original fluence map must be equal to the desired deliverable resolution, $\Delta y$.

The superposition of the two MLC fluence maps approximates the original fluence, as seen in Fig. 1. The MLC generated dose profile may then differ significantly from the desired dose. To examine these dose differences, a clinical fluence map produced by the Cadplan/Helios RTPS (Varian Medical Systems, Palo Alto, CA) derived for a six field parotid treatment was utilized. The resolution of this fluence is $0.5 \mathrm{~cm}$ in the $y$-direction across the leaves and $0.25 \mathrm{~cm}$ in the $x$-direction. This fluence was convolved with a scatter kernel to approximate the delivered dose. The kernel utilized was a two-dimensional Gaussian function with $\sigma=2 \mathrm{~mm}$,

$$
G(r)=\exp \left(-r^{2} / 2 \sigma^{2}\right) .
$$

The component fluence maps with a $1.0 \mathrm{~cm}$ MLC leaf width and a $0.5 \mathrm{~cm}$ sampling distance were derived as above and combined to yield the summated fluence. This was then convolved with the scatter kernel, and the dose was compared to the desired dose.

\section{Superposition method}

Fluence maps are currently produced by the RTP system with intensity bins equal to the leaf width in the $y$-direction. It may be possible to exactly reproduce this fluence by superimposing two offset component fluences, each with twice the original leaf width. This is illustrated in Fig. 2. A segment of a column from an original fluence map $c_{i, j}$ is shown where $i=1, \ldots, n$ with $n$ being the number of intensity bins in the $y$-direction, and $j=1, \ldots, m$ with $m$ being the number of intensity bins in the $x$-direction. Two component fluence maps, with intensity bins of twice the original width in the $y$-direction, are to be combined to reproduce the original 
intensity. These maps are denoted as $a_{k, j}$, and $b_{k, j}$ where $k$ $=1, \ldots, n / 2$ ( $n$ is an even number), and $j=1, \ldots, m$. This results in a series of linear equations to derive the intensities in the component maps. For the $j$ th column

$$
\begin{aligned}
& c_{1, j}=a_{1, j}+b_{1, j}, \\
& c_{2, j}=a_{2, j}+b_{1, j} \\
& \vdots \\
& c_{n-1, j}=a_{n / 2, j}+b_{n / 2-1, j}, \\
& c_{n, j}=b_{n / 2, j} .
\end{aligned}
$$

Consider bins $c_{1, j}$ and $c_{2, j}$ in the original map. It is clear that $b_{1, j}$ adds an offset intensity to these bins, and that the difference between $a_{2, j}$ and $a_{1, j}$ must be equal to the difference between $c_{1, j}$ and $c_{2, j}$. This gives

$$
\begin{aligned}
& a_{2, j}-a_{1, j}=c_{2, j}-c_{1, j}, \\
& a_{3, j}-a_{2, j}=c_{4, j}-c_{3, j} \\
& \vdots \\
& a_{n / 2, j}-a_{n / 2-1, j}=c_{n-2, j}-c_{n-3, j},
\end{aligned}
$$

$$
b_{n / 2, j}-b_{n / 2-1, j}=c_{n-1, j}-c_{n-2, j} .
$$

This yields equations for the relative intensities of the two component maps,

$$
\begin{aligned}
& a_{2, j}=a_{1, j}+c_{2, j}-c_{1, j}, \\
& a_{3, j}=a_{2, j}+c_{4, j}-c_{3, j} \\
& \vdots
\end{aligned}
$$

and

$$
\begin{aligned}
& b_{2, j}=b_{1, j}+c_{3, j}-c_{2, j}, \\
& b_{3, j}=b_{2, j}+c_{5, j}-c_{4, j} \\
& \vdots .
\end{aligned}
$$

The absolute intensities are found from $b_{n / 2, j}=c_{n, j}$. Alternatively $c_{1, j}$ will be zero, for typical clinical intensity maps, and this leads to $a_{1, j}=b_{1, j}=0$, which also enables calculation of the absolute intensities.

Figure 3(a) shows the column from the previous clinical intensity map with $0.5 \mathrm{~cm}$ fluence bins, and the component intensities that were calculated with $1.0 \mathrm{~cm}$ intensity bins. These summate to exactly reproduce the original fluence, however negative undeliverable intensities are required. To avoid these, the original fluence map must be filtered. A method was utilized where dummy values of the component intensites were calculated and if these were negative the original fluence was modified so that the (previously negative) component intensity would be zero. The component

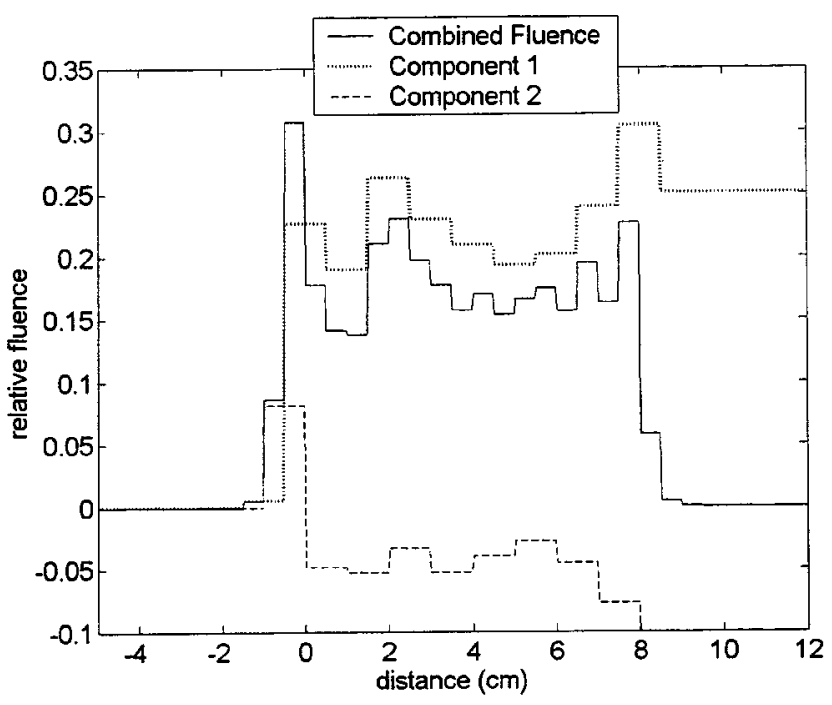

(a)

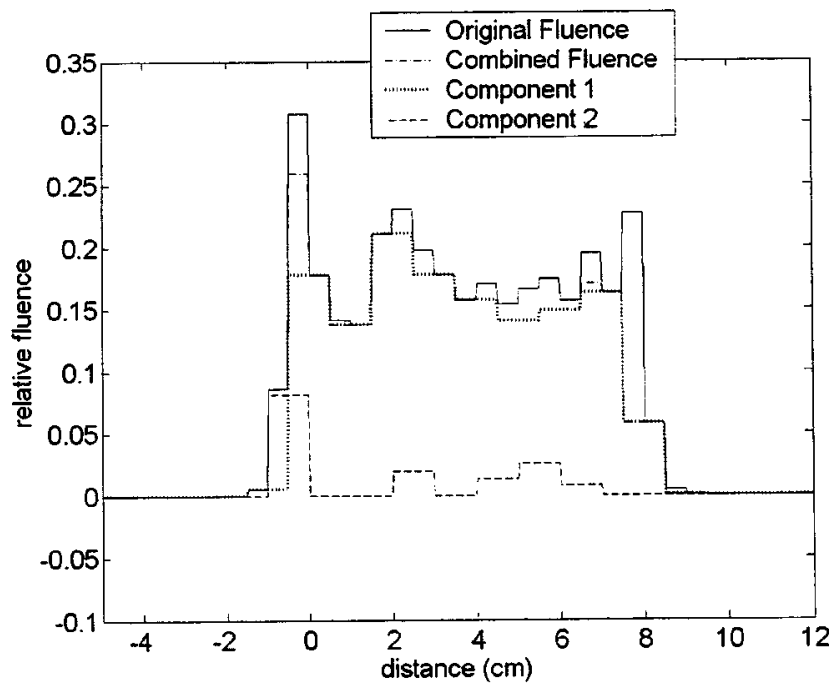

(b)

FIG. 3. (a) Profiles in the $y$-direction of the component fluence maps that combine to exactly reproduce the original fluence. Note that negative intensities result in one of the component maps. (b) Component map profiles where the original fluence map was filtered to remove negative intensities resulting in the component maps.

intensites were then calculated with the filtered fluence. Figure 3(b) shows the original fluence, the filtered fluence (sum of the non-negative component intensities) and the component maps. The difference between the filtered fluence and the desired fluence can be seen. The dose resulting from the combined or filtered fluence was calculated and compared to the dose from the original fluence map.

\section{B. Effect of sampling distance on delivered dose}

Initial investigations of the above methods resulted in large differences in fluence with the superposition method. The efficacy of the sampling method was experimentally in- 
vestigated. The effect of reducing the MLC leaf sampling distance on DMLC-IMRT distributions was examined for both 1.0 and $0.5 \mathrm{~cm}$ MLC leaf widths. This was investigated with a bar-pair pattern, an annular shaped intensity distribution, and quantitatively with a step-edge intensity distribution.

The following procedure was utilised to obtain dose distributions for the $1 \mathrm{~cm}$ MLC leaves, with $1.0 \mathrm{~cm}, 0.5 \mathrm{~cm}$, and $0.25 \mathrm{~cm}$ sampling distance, and the $0.5 \mathrm{~cm}$ MLC leaves with 0.5 and $0.25 \mathrm{~cm}$ sampling distance. High resolution test fluence maps were created by an in-house program with 0.25 $\mathrm{cm}$ resolution in the $y$-direction. For the $1 \mathrm{~cm}$ MLC leaves the high-resolution fluence map was split into four 0.25 $\times 1.0 \mathrm{~cm}$ fluence maps, each submap consisting of every fourth row of the original. These submaps were then converted to the fluence file format of the Cadplan/Helios RTPS. Leaf sequences were derived by Helios with the sliding window delivery method ${ }^{5,6}$ for each submap. We delivered one treatment with a single sequence. We then delivered a second treatment with two sequences offset by $0.5 \mathrm{~cm}$. Finally a third treatment was delivered with all four sequences offset by $0.25 \mathrm{~cm}$. The monitor units for each delivery were adjusted to give the same total dose to the film. These were recorded on Kodak XV film (Eastman-Kodak, Rochester, NY) placed at $1.5 \mathrm{~cm}$ depth in solid water (RMI, Middleton, WI) with a $6 \mathrm{MV}$ beam from a Varian 21EX accelerator (Varian Medical Systems, Palo Alto, CA). The films were digitized with $0.36 \mathrm{~mm}$ resolution using a Vidar VXR12 scanner (Vidar Systems, Herndon, PA). These were then normalized, the background level was subtracted, and sensitometric corrections were applied.

For the $0.5 \mathrm{~cm}$ MLC leaves the high-resolution fluence map was split into two $0.25 \times 0.5 \mathrm{~cm}$ optimal fluence maps. Leaf sequences were calculated for each. A single leafsequence was delivered to film, as well as sequential delivery with the couch offset by $0.25 \mathrm{~cm}$ between each sequence.

\section{Bar-pattern}

A bar-pattern fluence map with $0.25 \times 0.25 \mathrm{~cm}$ resolution was developed, consisting of bar-pairs with gradually reducing bar-width and spacing (Fig. 4). The bars were oriented obliquely at an angle of $14^{\circ}$ to the leaf-motion direction, so that the effect on resolution in the direction perpendicular to leaf motion could be examined. The bar-pairs consisted of bars of approximately $3.7,2.3,1.8,1.4$, and $0.9 \mathrm{~cm}$ centerto-center distance. This translates to $0.27,0.43,0.56,0.7$, and 1.1 line-pairs per $\mathrm{cm}$. The relative intensity was set to 1.0 for the bars, and 0.2 for the interbar spaces.

\section{Annular intensity distribution}

To further investigate the resolution of dose delivery with the MLC sampling distance, an annular or "donut" shaped intensity distribution was created. This pattern consisted of a $3.5 \mathrm{~cm}$ diameter inner circle of low intensity surrounded by an annular high intensity region of $11.5 \mathrm{~cm}$ outer diameter. The inner circle was assigned a relative intensity level of 0.2 while the outer annulus was assigned a relative intensity level of 1 . The areas outside of the annulus were assigned an

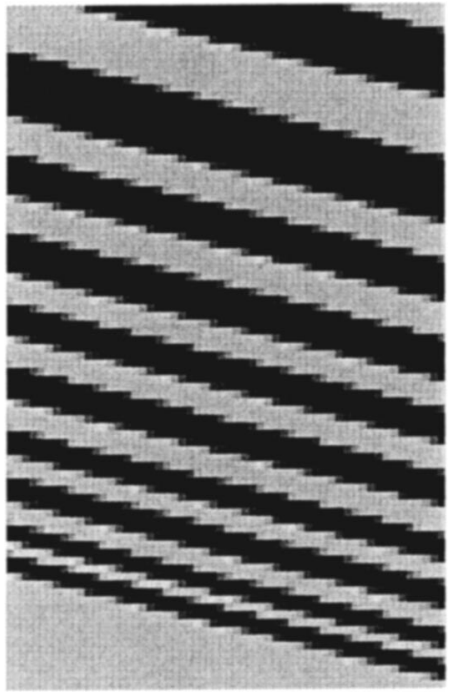

FIG. 4. Bar-pair fluence distribution to examine the resolution in the direction perpendicular to leaf motion. The bar-pairs consist of approximately $0.3,0.4,0.6,0.7$, and 1.1 line-pairs per $\mathrm{cm}$. The relative intensity was set to 1.0 for the bars, and 0.2 for the interbar spaces.

intensity value of 0.2 . This tests the ability of the dosedelivery system to deliver a specifically shaped dose region, requiring high resolution in directions both parallel and perpendicular to leaf motion.

\section{Edge distributions}

To obtain a quantitative measure of the resolution in the $y$-direction perpendicular to leaf motion with sampling distance, a step-edge distribution was created. This consisted of a step-edge in intensity angled at 14 degrees to the leaf motion direction. One side of the step-edge was assigned a relative intensity level of 1 and the other side was assigned an intensity level of 0.2 . This pattern was designed to test the ability of the MLC delivery to define different dose regions in the direction perpendicular to leaf motion.

To enable comparison with the inherent limiting resolution obtainable with this MLC design for DMLC-IMRT, the step-edge was also formed with the edge aligned perpendicular to the leaf motion direction. In this direction the resolution of the IMRT delivery is determined by the limiting penumbra of the MLC leaf-ends, in-phantom scatter, and the MLC system leaf-speed and dose rate control.

Films of the irradiations were measured and digitized as described earlier. To analyze the dose-distributions at the edge, the "effective penumbra" and "dose-undulation" metrics developed for static MLC edges were utilized. ${ }^{7}$ To quantify the effective penumbra, tangents to the inner peaks of the $80 \%$ and the outer peaks of the $30 \%$ isodose contours were drawn and the perpendicular distance between these measured. Similarly for the dose undulation, tangents were drawn to the inner and outer peaks of the $60 \%$ isodose contour, and the perpendicular distance was measured. For static fields, the penumbra is normally quantified by $80-20 \%$ width and the undulation by the $50 \%$ isodose. However, for these IMRT fields, the base intensity was set to $20 \%$ rather 


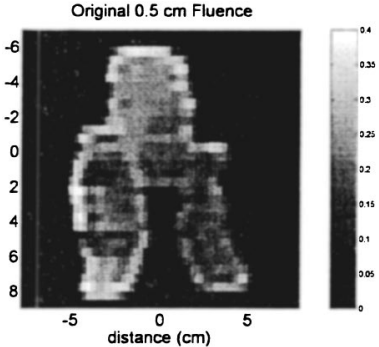

(a)

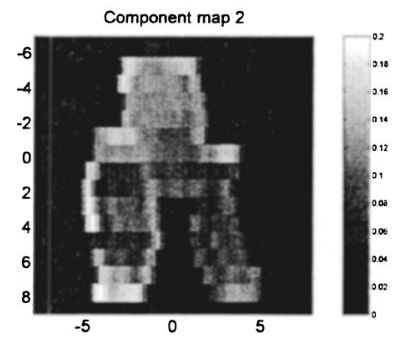

(c)

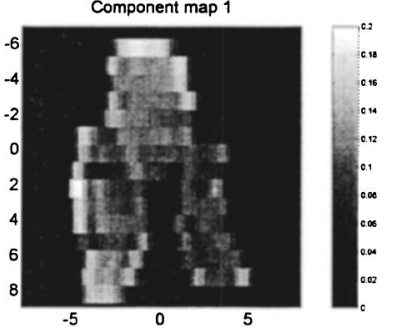

(b)

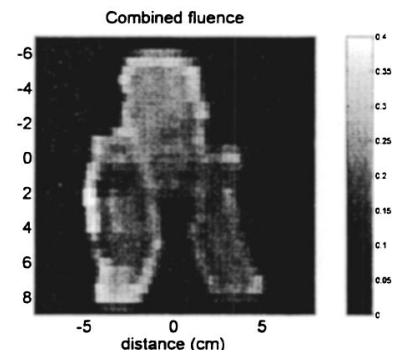

(d)

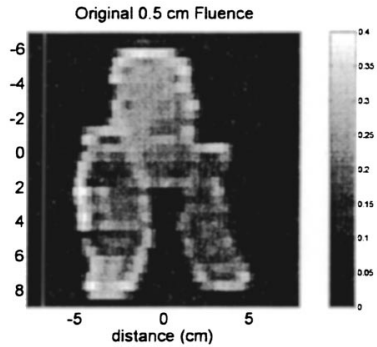

(a)

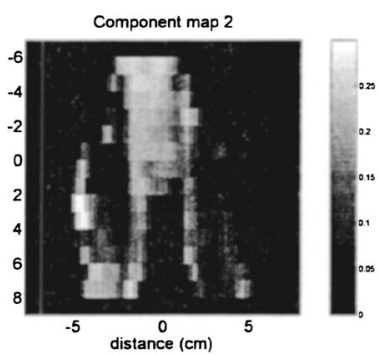

(c)

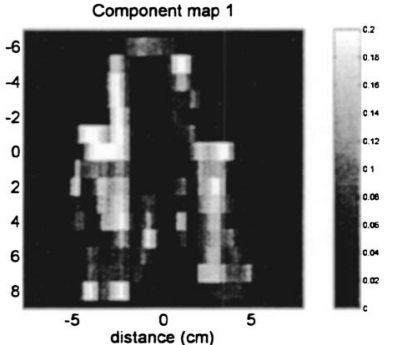

(b)

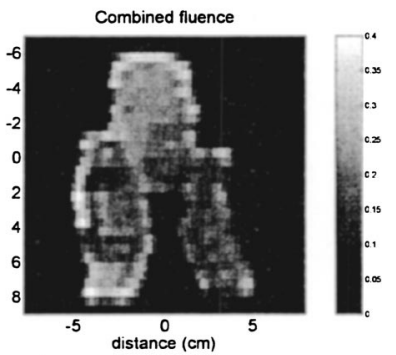

(d)

FIG. 5. Example of the sampling method applied to a clinical fluence map for a field from a six field parotid plan derived for a MLC leaf width of $L$ $=0.5 \mathrm{~cm}$. (a) Original fluence map with $0.5 \mathrm{~cm}$ bins. (b) and (c) two component maps with $1.0 \mathrm{~cm}$ bins. (d) Resulting combined fluence from the two components.

than zero. Therefore the $80-30 \%$ distance and the $60 \%$ isodose line were chosen to give representative values for these metrics.

\section{RESULTS}

\section{A. Method to reduce the sampling distance for DMLC-IMRT}

\section{Sampling method}

The original clinical fluence from the parotid plan with $0.5 \mathrm{~cm}$ resolution in the $y$-direction is shown in Fig. 5(a).

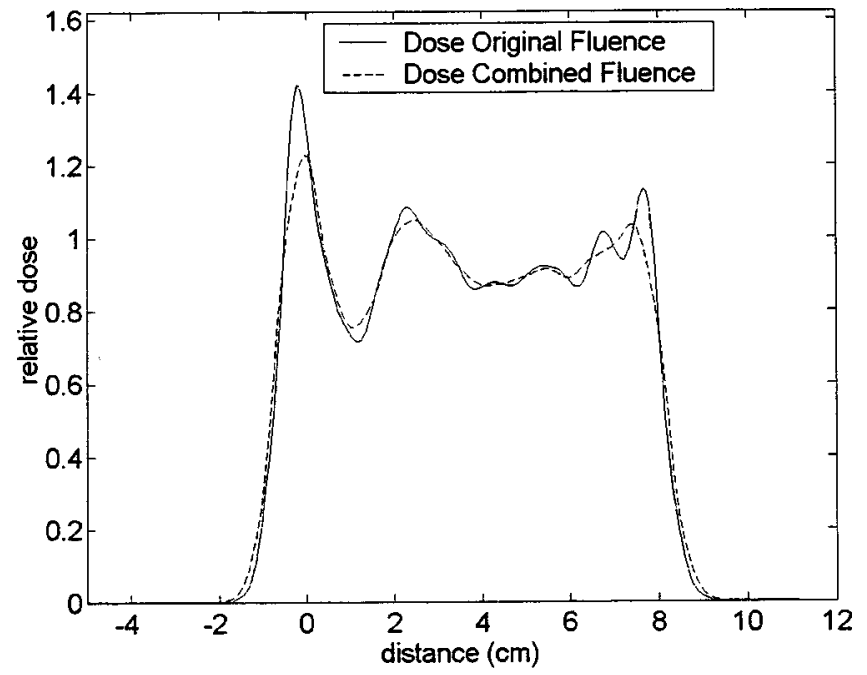

FIG. 6. Dose profiles in the $y$-direction from the combined fluence formed by two $1.0 \mathrm{~cm}$ leaf width MLC irradiations and the sampling method, compared to the desired dose from the original $0.5 \mathrm{~cm}$ fluence map.
FIG. 7. Example of the superposition method applied to the clinical fluence map. (a) Original fluence map with $0.5 \mathrm{~cm}$ bins; (b) and (c) two component maps with $1.0 \mathrm{~cm}$ bins, (d) resulting combined fluence from the two components.

The component intensity maps, each with $1.0 \mathrm{~cm}$ leaf width, and the resulting combined fluence are also shown in Fig. 5. A profile from the dose map calculated from the combined fluence compared to the dose from the original fluence is shown in Fig. 6. The same profile illustrated in Fig. 1 is used. The resulting difference in dose is smaller than the difference in fluence. The differences could potentially be decreased further by deconvolving the original fluence with an inverse filter that represents the blur introduced by the larger leaf

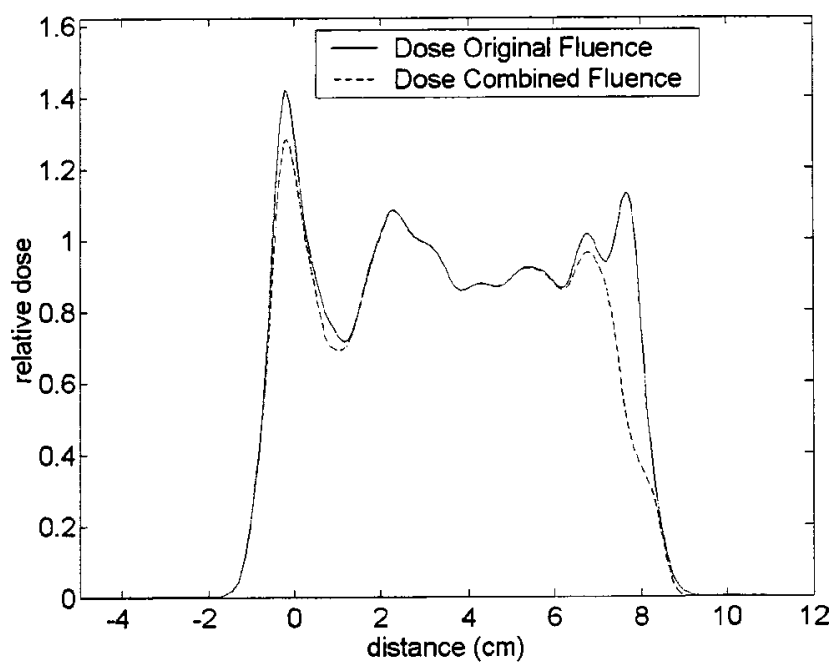

FIG. 8. Dose profiles in the $y$-direction from the combined fluence formed by two $1.0 \mathrm{~cm}$ leaf width MLC irradiations and the superposition method, compared to the desired dose from the original $0.5 \mathrm{~cm}$ fluence map. 

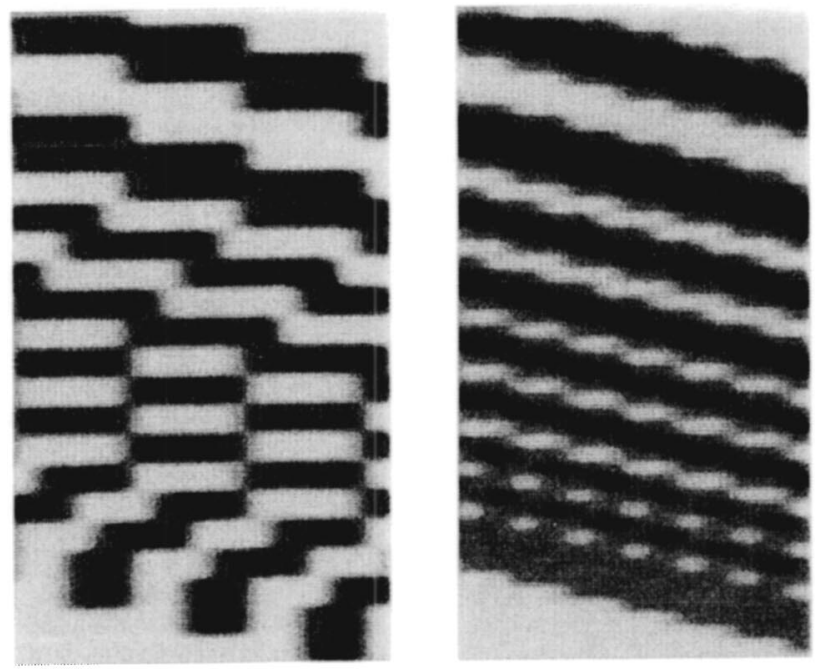

(a)

(b)

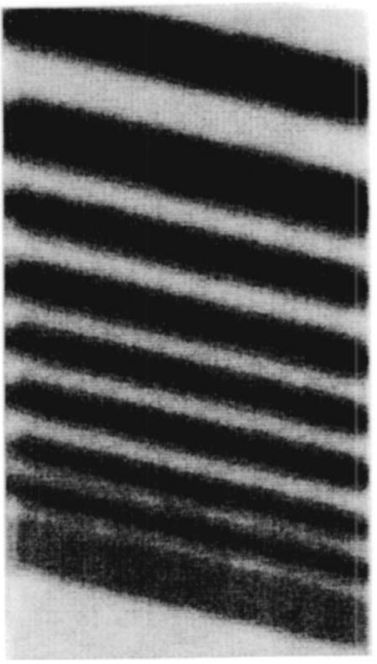

(c)

FIG. 9. Films of the bar-pattern IMRT irradiations for the $L=1.0 \mathrm{~cm}$ MLC. The results are shown for (a) one segment with $\Delta=1.0 \mathrm{~cm}$ sampling distance, (b) two segments with $\Delta=0.5 \mathrm{~cm}$, and (c) four segments with $\Delta$ $=0.25 \mathrm{~cm}$ sampling distance. These show that the highest frequency barpattern is not resolved with this leaf width. The bar-patterns become more clearly defined with reducing sampling distance.

width. ${ }^{9}$ Further investigation is underway to examine this before the application of this method to clinical IMRT fields becomes possible.

\section{Superposition method}

Figure 7 shows the component intensity maps calculated by this method from Eq. (5) with $a_{1, j}=b_{1, j}=0$. The component maps were calculated by first filtering the desired fluence so that negative intensities in the component maps would not occur. The filtered fluence is equal to the combined fluence that is shown in Fig. 7(d). This has a similar appearance to the original fluence, however some large differences in the fluence can occur as seen in Fig. 3(b). The

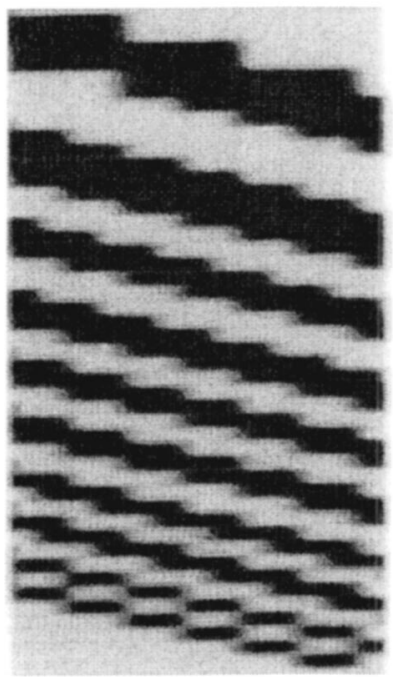

(a)

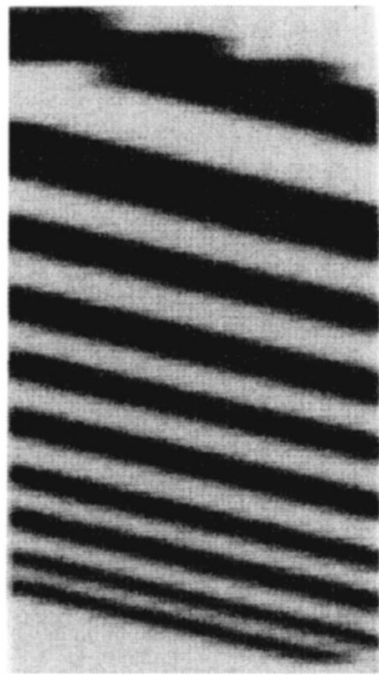

(b)
FIG. 10. Films of the bar-pattern IMRT irradiations for the MLC with $L$ $=0.5 \mathrm{~cm}$ leaf width for (a) one segment with $\Delta=0.5 \mathrm{~cm}$ sampling distance, (b) two segments with $\Delta=0.25 \mathrm{~cm}$. All bar-patterns are resolved; however, the $\Delta=0.25 \mathrm{~cm}$ result does not exhibit noticeable stair-stepping and more closely resembles the original bar-pattern fluence.

dose resulting from the combined fluence compared to the dose from the original fluence is shown in Fig. 8 for the same column of the fluence map utilized previously. This demonstrates that large differences in dose can result with this method. However, where fluence differences between the combined and original fluence maps are large, the component maps could be further modified to minimize the fluence difference. Further investigation of this method is currently underway to determine whether this is feasible.

\section{B. Effect of sampling distance on delivered dose}

\section{Bar-patterns}

The film images resulting from the irradiations of the barpair fluence for the $1.0 \mathrm{~cm}$ MLC are shown in Fig. 9. The MLC leaf motion was from left-to-right in the figure. The figure shows results for sampling distances of (a) $1.0 \mathrm{~cm}$, (b) $0.5 \mathrm{~cm}$, and (c) $0.25 \mathrm{~cm}$. These demonstrate a marked improvement in the appearance of the bar-pairs with decreasing MLC sampling distances. For the $1.0 \mathrm{~cm}$ sampling distance most of the bar-pairs are not identifiable as bars, presenting as a checkerboard appearance. The highest frequency barpair $(1.1 \mathrm{lp} / \mathrm{cm})$ is not resolved with this leaf width, even at the smaller sampling distances. The edges of the bars are also quite blurred with decreasing leaf sampling distances due to the relatively large leaf width.

Figure 10 shows the bar-pattern results for the $0.5 \mathrm{~cm}$ MLC leaf width for (a) $0.5 \mathrm{~cm}$ sampling distance, and (b) $0.25 \mathrm{~cm}$ sampling distance. The smaller sampling distance improves the appearance of the bars, and the image closely resembles the original fluence (Fig. 4). All the bar-pairs are resolved with this leaf width, although the $0.5 \mathrm{~cm}$ sampling distance has a very stepped appearance for the higher fre- 


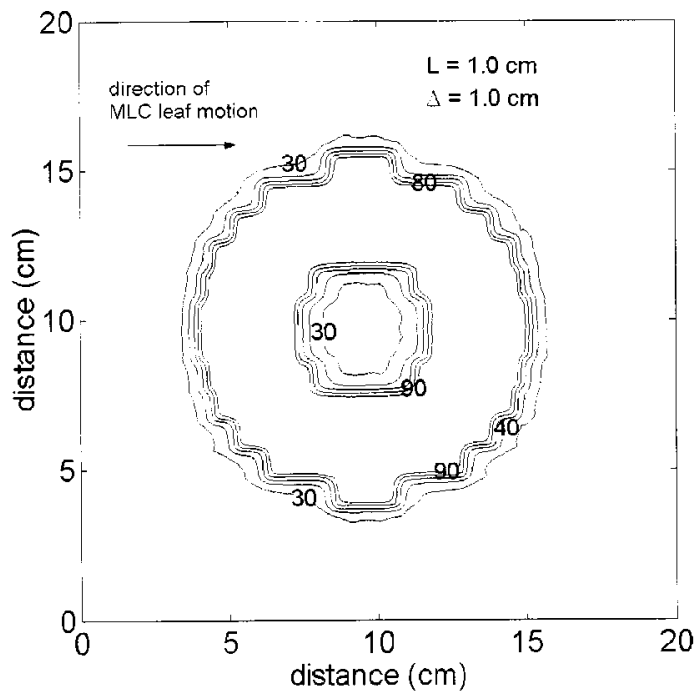

(a)

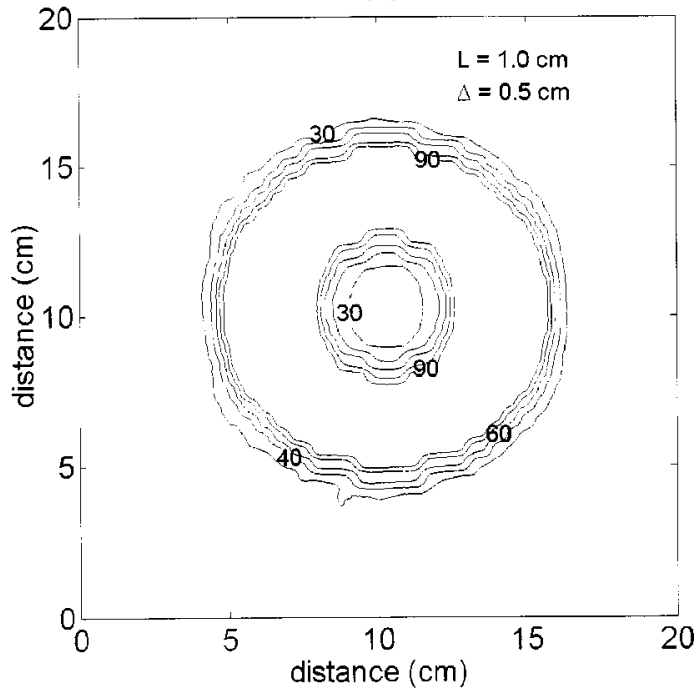

(b)

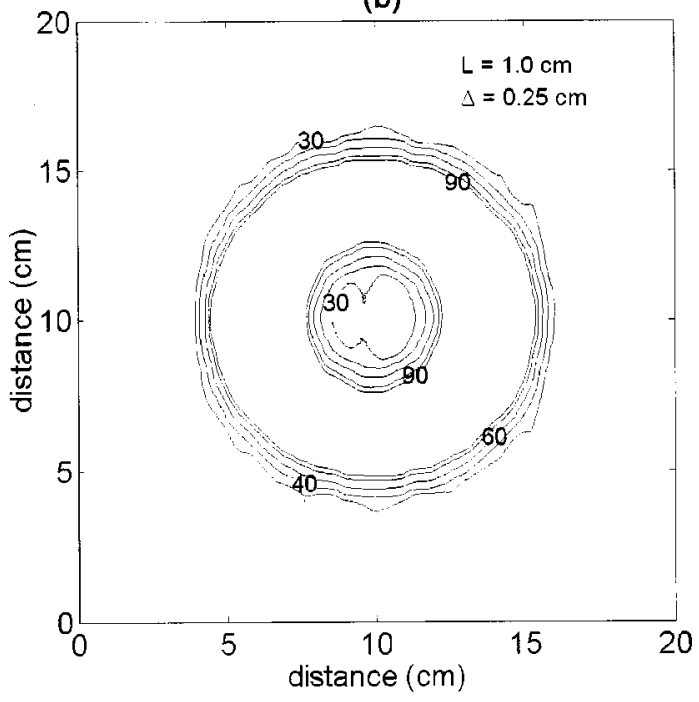

(c)

FIG. 11. Isodose distributions for the annular or "donut" shaped intensity distribution delivered with a MLC with $L=1.0 \mathrm{~cm}$ leaf width for leaf sampling distances $\Delta$ of (a) $1.0 \mathrm{~cm}$, (b) $0.5 \mathrm{~cm}$, and (c) $0.25 \mathrm{~cm}$. These results demonstrate improvement of the shaping of the intensity pattern with decreasing leaf sampling distance. The dose falls off more slowly in the direction perpendicular to leaf motion due to the relatively large leaf width.

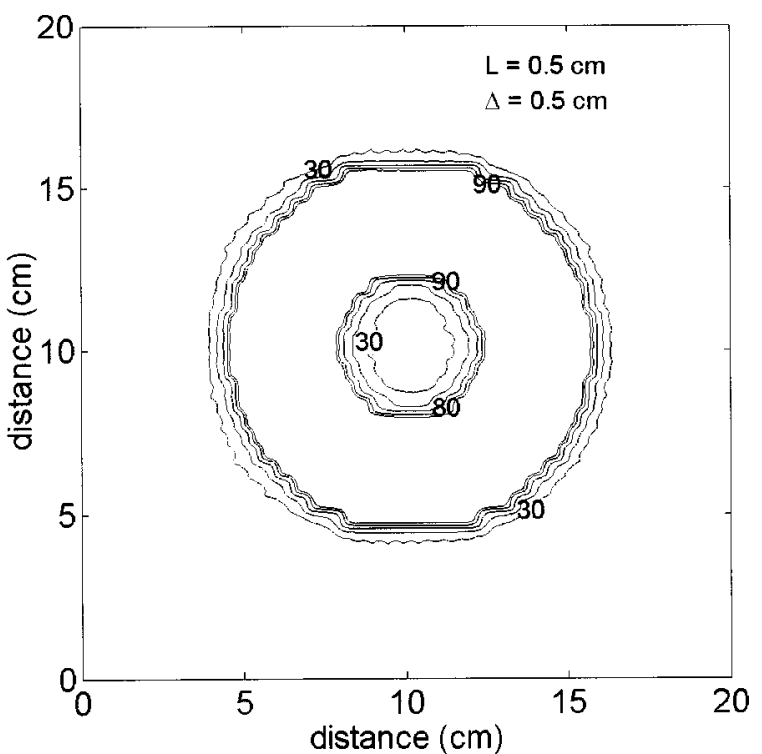

(a)

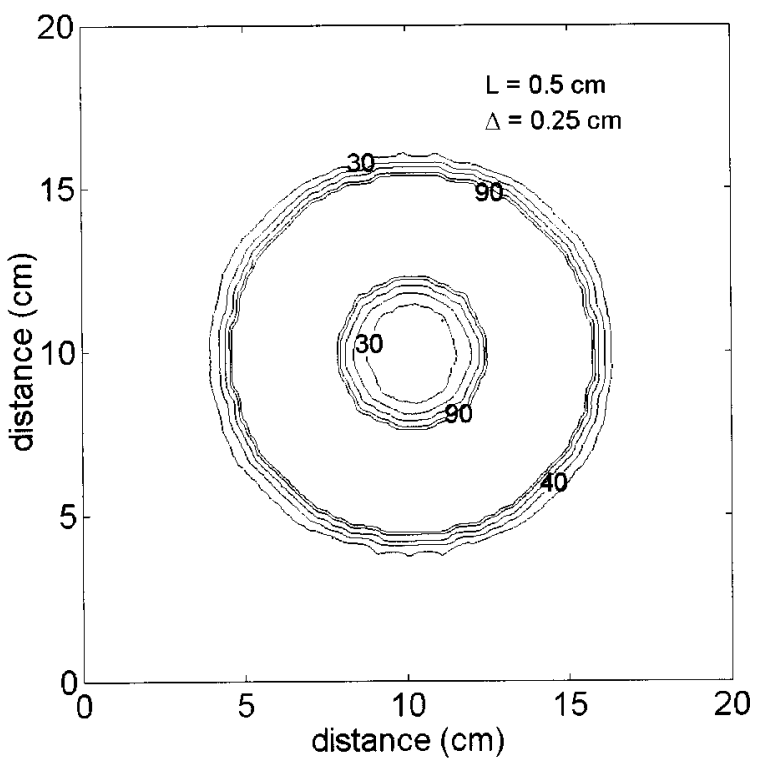

(b)

FIG. 12. Isodose distributions for the annular or "donut" shaped intensity distribution delivered with a MLC with $L=0.5 \mathrm{~cm}$ leaf width for leaf sampling distances $\Delta$ of (a) $0.5 \mathrm{~cm}$, and (b) $0.25 \mathrm{~cm}$. The $0.25 \mathrm{~cm}$ sampling distance results in conformation or shaping of the dose that is independent of the direction.

quency bars. The more stepped edge at the top of the figure is due to $1.0 \mathrm{~cm}$ MLC leaves. The central $20 \mathrm{~cm}$ of the MLC is made of 40 leaf pairs with a width of $0.5 \mathrm{~cm}$, while the remaining outer leaves have a width of $1.0 \mathrm{~cm}$.

\section{Annular intensity distribution}

The annular or "donut" shaped intensity distributions formed by the dynamic IMRT delivery for the $1.0 \mathrm{~cm}$ MLC leaf width are shown in Fig. 11 for sampling distances $\Delta$ of (a) $1.0 \mathrm{~cm}$, (b) $0.5 \mathrm{~cm}$, and (c) $0.25 \mathrm{~cm}$. The undulation is reduced with decreasing sampling distance. For the $\Delta$ $=1.0 \mathrm{~cm}$ result, only the $30 \%$ isodose is not highly undulat- 


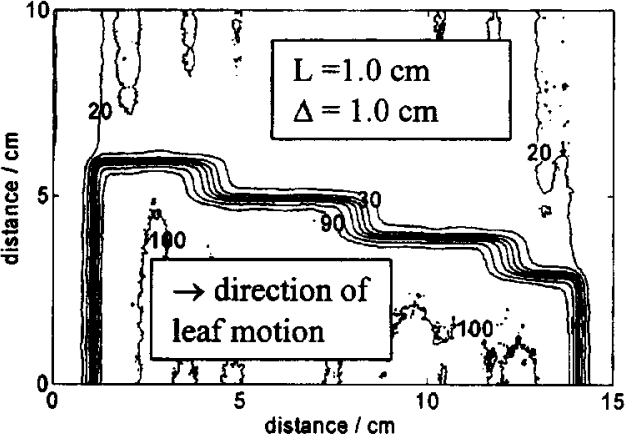

(a)

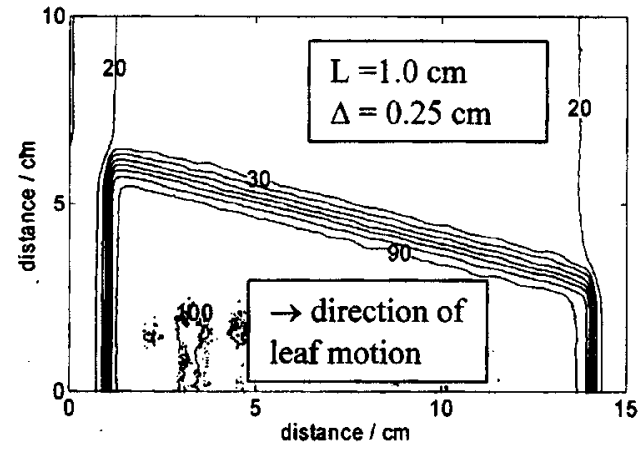

(c)

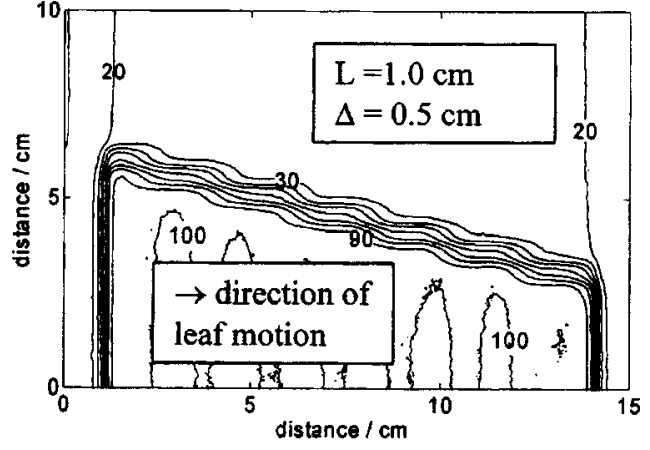

(b)

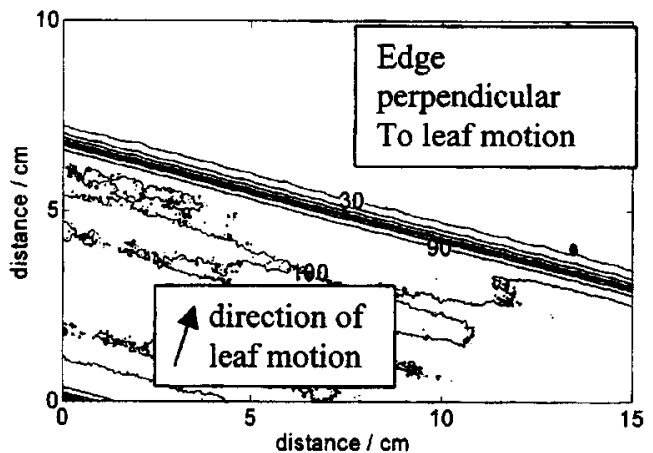

(d)

FIG. 13. Dose gradient in the direction perpendicular to leaf motion with sampling distance for the $1.0 \mathrm{~cm}$ MLC leaf width. Edge dose distributions for the $1.0 \mathrm{~cm}$ leaf width for (a) $1.0 \mathrm{~cm}$ sampling distance, (b) $0.5 \mathrm{~cm}$ sampling distance, and (c) $0.25 \mathrm{~cm}$ sampling distance. The result for the edge aligned perpendicularly to the direction of leaf-motion is shown in (d). This demonstrates the dose gradient in the direction of leaf motion and the limiting dose resolution of the MLC.

ing, whereas for the $\Delta=0.25 \mathrm{~cm}$ result the undulation is removed. The dose gradient is lower in the $y$-direction than in the $x$-direction. For the $\Delta=0.25 \mathrm{~cm}$ result the width of the $80-30 \%$ isodose can be seen to increase from the side to the top/bottom of the distribution. This width could be measured at a sample of angles ${ }^{8}$ to quantify the differences; however, a quantitative comparison of the resolution in the direction perpendicular to leaf motion is made in the next section for an edge intensity distribution.

The results for the $0.5 \mathrm{~cm}$ MLC leaf width are shown in Fig. 12 for sampling distances of (a) $\Delta=0.5 \mathrm{~cm}$ and (b) $\Delta$ $=0.25 \mathrm{~cm}$. The dose conformation is superior to that obtained with a larger leaf width. The $\Delta=0.25 \mathrm{~cm}$ sampling distance removes the undulation and results in conformation or shaping of the dose that is virtually independent of direction.

\section{Edge distribution}

Figure 13 shows the measured isodose distributions for the step-edge with $1.0 \mathrm{~cm}$ leaf width. The distributions are shown for sampling distances of (a) 1.0, (b) 0.5, and (c) 0.25 $\mathrm{cm}$. For comparison, the distribution for the step-edge formed with the edge perpendicular to the leaf-motion direction is shown in (d). This edge distribution is displayed at a similar angle to the other results to facilitate visual compari- son. The measured $80-30 \%$ effective penumbra and the dose undulation of the $60 \%$ isodose line are given in Table I. The penumbra is reduced by approximately $2 \mathrm{~mm}$, for the $0.5 \mathrm{~cm}$ and $0.25 \mathrm{~cm}$ sampling distances. The undulation is small for the $0.5 \mathrm{~cm}$ sampling distance and removed for the $0.25 \mathrm{~cm}$ sampling distance. These results suggest that the improvement for sampling distances below $0.5 \mathrm{~cm}$ is small. The dose gradient is much less steep than that obtained with the edge perpendicular to the leaf-motion direction, the 80-

TABLE I. Results for the oblique intensity step-edge formed by the dynamic IMRT delivery to quantify the resolution in the direction perpendicular to leaf motion. Measured 80-30\% effective penumbra and dose undulation of the $60 \%$ isodose line are given for the two MLC leaf widths and the reduced leaf sampling distances.

\begin{tabular}{lcccccc}
\hline \hline $\begin{array}{l}\text { Leaf width } \\
\text { /cm }\end{array}$ & \multicolumn{2}{c}{1.0} & & & 0.5 & Straight \\
\hline $\begin{array}{l}\text { Sampling } \\
\text { distance/ } \\
\mathrm{cm}\end{array}$ & 1.0 & 0.5 & 0.25 & 0.5 & 0.25 & \\
$\begin{array}{l}80-30 \% \\
\text { Penumbra/ } \\
\text { mm }\end{array}$ & 13 & 10.8 & 10.8 & 8.5 & 7.0 & 6.2 \\
$\begin{array}{l}\text { Undulation } \\
\text { /mm }\end{array}$ & 7.0 & 2.0 & 0.0 & 2.8 & 0.0 & 0.0 \\
\hline \hline
\end{tabular}




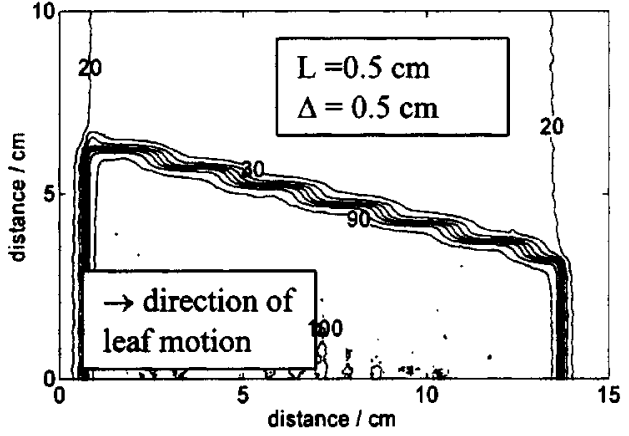

(a)

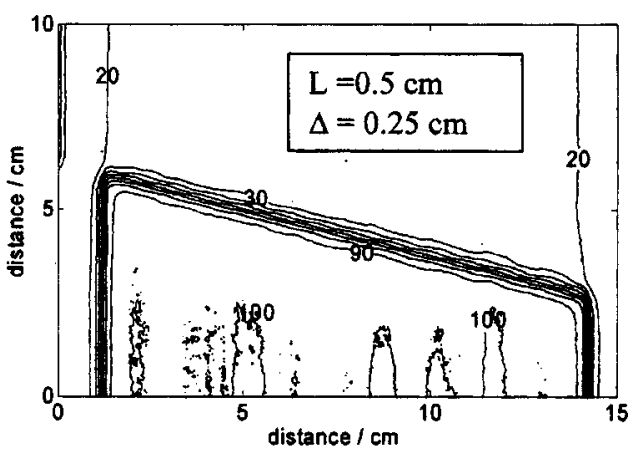

(b)
$30 \%$ distance being $7 \mathrm{~mm}$ greater for the $1.0 \mathrm{~cm}$ sampling distance and $5 \mathrm{~mm}$ greater for the smaller sampling distances.

The results for the $0.5 \mathrm{~cm}$ MLC leaf width are similarly shown in Fig. 14 for sampling distances of (a) $0.5 \mathrm{~cm}$ and (b) $0.25 \mathrm{~cm}$, and the quantitative results are given in Table I. These results show that the dose gradient is steeper for the $0.5 \mathrm{~cm}$ MLC leaf width than for the $1.0 \mathrm{~cm}$. With the 0.25 $\mathrm{cm}$ sampling distance the dose gradient is similar to that for the straight edge perpendicular to the direction of leafmotion, and there is no undulation.

\section{DISCUSSION}

The two methods examined here both show promise in improving the resolution of clinical IMRT delivery. These were applied to the specific case of utilizing a $1.0 \mathrm{~cm}$ leaf width MLC to reproduce a clinical fluence map with $0.5 \mathrm{~cm}$ intensity bins. However, further work is necessary to determine the accuracy with which clinical fluence and dose can be produced with these methods. For the sampling method, the use of inverse filtering ${ }^{9}$ of the desired fluence before calculation of the component deliveries requires investigation. Alternatively, overall dose comparisons for multiple field treatments with the original and combined fluences would be useful in determining the clinical effect of the dose deterioration. These could be compared to the dose calculated for the larger MLC leaf width without any reduction in sampling distance to quantify improvements in clinical IMRT distributions obtained by utilizing this method. For the superposition method, a means of reducing the large fluence differences observed here is needed. To further study these methods, clinical fluence maps with high resolution would be utilized. Currently these are difficult to produce, as RTP systems generally produce maps with leaf width resolution in the $y$-direction. For example, the Helios system produces clinical fluence maps with $0.25 \mathrm{~cm}$ bins in the $x$-direction and $0.5 \mathrm{~cm}$ (for $0.5 \mathrm{~cm}$ leaf width MLC) in the $y$-direction.

The $1.0 \mathrm{~cm}$ MLC was clearly able to resolve more linepairs with reduction of the sampling distance to $0.5 \mathrm{~cm}$. However the highest frequency $(1.1 \mathrm{lp} / \mathrm{cm})$ was not resolved for this leaf width, but was clear with the $0.5 \mathrm{~cm}$ MLC. In these experiments the sampling distance in the $y$-direction was varied while the sampling distance or intensity bin size in the $x$-direction was set to $0.25 \mathrm{~cm}$ for all cases. This is fixed within the Varian leaf motion calculator and MLC system and could not be varied. Whether this sampling distance is sufficient to produce an arbitrary continuous dose profile in the $x$-direction is unknown. This sampling distance may affect how well the bar-patterns are reproduced; however, its effect is the same for all leaf widths and sampling distances utilized.

These results show that the production of an arbitrarily shaped intensity distribution is improved by reducing the sampling distance. The utilization of micro or mini multileaf collimators for small field or stereotactic IMRT is increasing. However, these multileaf collimators are expensive "addon" devices and can only produce relatively small field apertures. Conventional MLCs are now standard equipment on linear accelerators. The results here for the $0.5 \mathrm{~cm}$ MLC leaf width with a sampling distance of $0.25 \mathrm{~cm}$ suggest that investigation of this method for stereotactic IMRT is warranted.

The step-edge distributions provide a means of quantifying the dose gradient for IMRT in the direction perpendicular to leaf motion with varying MLC leaf sampling distance. An edge at a small angle was utilized so that the dose gradient measured was not simply that of the tongue-and-grooved leaf-side. These results show that a $0.5 \mathrm{~cm}$ sampling distance was sufficient to obtain the best achievable resolution with the $1.0 \mathrm{~cm}$ MLC. Although some undulation remained, the overall dose gradient was the same as the $0.25 \mathrm{~cm}$ sampling distance. For the $0.5 \mathrm{~cm}$ MLC leaf width, the dose gradient is significantly improved over the $1.0 \mathrm{~cm}$ MLC and there is relatively small undulation. With the $0.5 \mathrm{~cm}$ leaf design and a sampling distance of $0.25 \mathrm{~cm}$, the resolution of the DMLC-IMRT delivery is nearly independent of direction.

The implications for IMRT optimization algorithms are significant in that the optimization must be performed with the higher resolution or smaller voxel and pencil-beam size. Further work is required to bring this concept to a clinically viable system. In the case of fields with the collimator angled or nonaxial radiotherapy fields, three-dimensional couch movement is required to move the patient in the direction perpendicular to the MLC leaf motion. The secondary collimator jaws defining the edges of the field (along the leaf motion direction) would also have to move by the same dis- 

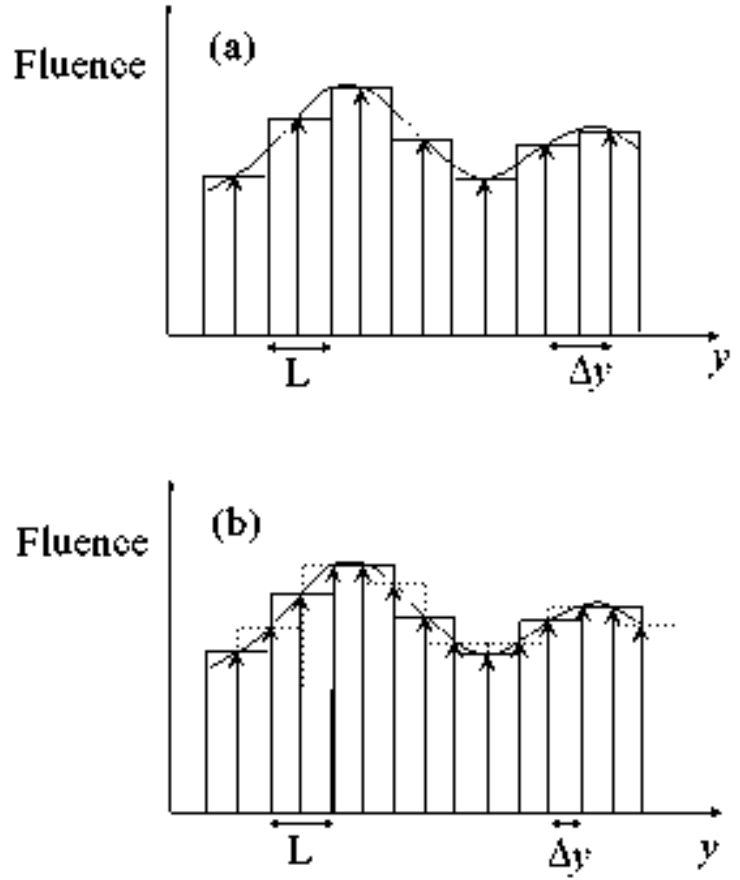

FIG. 15. Illustration of the concept of MLC sampling distance as applied to intensity modulated radiation therapy. In (a) the motion of each MLC leafpair is considered to produce a rectangular intensity bin of leaf width $L$, and the sampling distance $\Delta$ is the leaf center-to-center distance. By combining a second MLC motion offset by half the leaf width as seen in (b), the sampling distance can be reduced to $\Delta=L / 2$.

tance as the couch offset to maintain the field aperture relative to the patient anatomy. Accurate couch motion in all directions is possible with modern accelerators and has been commercially implemented for static field therapy. The Siemens HD270 (Siemens Medical Systems, Concord, CA) approach to smoothing static MLC field edges is an example. The interleaf leakage radiation to a point will also be significantly reduced with this method. However this may not be important for treatments with several beams and at depth within the patient.

Bortfeld et al. ${ }^{9}$ theoretically examined the minimal or optimal leaf width of a multileaf collimator considering the inherent limiting dose-spread that occurs due to scatter. They determined that the sampling distance equals the 20\%-80\% penumbra divided by 1.7 . They utilized 2.5 to $3 \mathrm{~mm}$ for the penumbra for a $6 \mathrm{MV}$ beam and small fields to give 1.5-2 $\mathrm{mm}$ as the required sampling distance. They found that the leaf width could be twice the sampling distance with relatively small deterioration in the dose. The results here suggest that the use of $0.25 \mathrm{~cm}$ leaf sampling distance results in near optimal dose resolution for this $0.5 \mathrm{~cm}$ width MLC. Further improvement in dose delivery may be possible for this leaf width by reduction of the MLC penumbra with a double-focused design. Further measurements with smaller leaf widths and sampling distances are required to show whether dose resolution can be improved further. The effect of random setup variation and in-phantom scatter will also modify the delivered intensity.

\section{CONCLUSION}

The effect of reducing the leaf sampling distance on dose resolution for dynamic IMRT was examined for both 1.0 and $0.5 \mathrm{~cm}$ MLC leaf width utilizing bar-patterns, an annular shaped distribution and an intensity step-edge. Improvement in resolution was found for the $1.0 \mathrm{~cm}$ leaf width with a sampling distance of $0.5 \mathrm{~cm}$, with only a small benefit for further reduction. For the $0.5 \mathrm{~cm}$ leaf width, a sampling distance of $0.25 \mathrm{~cm}$ resulted in a resolution in the direction perpendicular to leaf motion similar to that in the leaf motion direction. The resolution of IMRT delivery with MLCs can be improved by reducing the leaf sampling distance and the proposed method is independent of the leaf sequencing algorithm.

\section{APPENDIX: MLC SAMPLING DISTANCE}

The theoretical effect of MLC leaf sampling distance on IMRT fluence and dose was discussed in detail by Bortfeld et al. ${ }^{9}$ To briefly illustrate this, the curve in Fig. 15(a) shows an arbitrary desired fluence profile $\Phi(y)$ in the direction perpendicular to leaf motion. The fluence map is considered to be at a sufficiently small sample spacing to be considered as a continuous function.

Figure 15(a) shows the normal situation where the leaf width is equal to the sampling distance. Each MLC leaf-pair produces a rectangular intensity bin of width equal to the leaf width $L$, ignoring penumbral and tongue and groove effects, with the sampling distance (distance between leaf centers) equal to $\Delta y$. The sampling distance is represented by the $\uparrow$ symbols and the rectangular fluence bin by the dotted lines. The resulting fluence from the MLC can be represented as ${ }^{9}$

$$
\Phi_{\mathrm{MLC}}(y)=\frac{1}{L}[\Phi(y) \operatorname{III}(y / \Delta y)] * \operatorname{rect}(y / L)
$$

where

$$
\operatorname{rect}(y)= \begin{cases}1 & \text { if }|y|<1 \\ 0 & \text { otherwise }\end{cases}
$$

and the sampling comb by

$$
\operatorname{III}(y)=\sum_{\mathrm{n}=-\infty}^{\infty} \delta(y-n) .
$$

The sampling distance $\Delta y$ can be reduced to half the leaf width by two irradiations with a couch shift of $\Delta y$ between them. This is illustrated in Fig. 15(b).

\footnotetext{
${ }^{a)}$ Now at Dept. of Radiation Oncology, Newcastle Mater Hospital, Newcastle, Australia; electronic mail: peter.greer@mater.health.nsw.gov.au

${ }^{1}$ P. M. Evans and M. Partridge, "A method of improving the spatial resolution of treatments that involve a multileaf collimator," Phys. Med. Biol. 45, 609-622 (2000).

${ }^{2}$ R. A. C. Siochi, "Virtual micro-intensity modulated radiation therapy," Med. Phys. 27, 2480-2493 (2000)

${ }^{3}$ M. E. Welch and T. J. Dacy, "Is high resolution obtainable on both the blade tip and the edge of the Phillips multileaf collimator. What techniques are required to achieve this?" Phys. Med. Biol. 39A, 496 (1994).
} 
${ }^{4}$ J. M. Galvin, D. D. Leavitt, and A. A. Smith, "Field edge smoothing for multileaf collimators," Int. J. Radiat. Oncol., Biol., Phys. 35, 89-94 (1996).

${ }^{5}$ D. J. Convery and M. E. Rosenbloom, "The generation of intensity modulated fields for conformal radiotherapy by dynamic collimation," Phys. Med. Biol. 37, 1359-1374 (1992).

${ }^{6}$ S. V. Spirou and C. S. Chui, "Generation of arbitrary intensity profiles by dynamic jaws or multileaf collimators," Med. Phys. 21, 1031-1041 (1994).
${ }^{7}$ J. M. Galvin, K. Han, and R. Cohen, "A comparison of multileaf collimator and alloy block field shaping," Int. J. Radiat. Oncol., Biol., Phys. 40, 721-731 (1998).

${ }^{8}$ J. H. Killoran, J. Y. Giraud, and L. Chin, "A dosimetric comparison of two multileaf collimator designs," Med. Phys. 29, 1752-1758 (2002).

${ }^{9} \mathrm{~T}$. Bortfeld, U. Oelfke, and S. Nill, "What is the optimum leaf width of a multileaf collimator?” Med. Phys. 27, 2494-2502 (2000). 